\title{
Control of progesterone receptor transcriptional synergy by SUMOylation and deSUMOylation
}

\author{
Hany A Abdel-Hafiz ${ }^{1,3^{*}}$ and Kathryn B Horwitz ${ }^{1,2}$
}

\begin{abstract}
Background: Covalent modification of nuclear receptors by the Small Ubiquitin-like Modifier (SUMO) is dynamically regulated by competing conjugation/deconjugation steps that modulate their overall transcriptional activity. SUMO conjugation of progesterone receptors (PRs) at the N-terminal lysine (K) 388 residue of PR-B is hormone-dependent and suppresses PR-dependent transcription. Mutation of the SUMOylation motif promotes transcriptional synergy.

Results: The present studies address mechanisms underlying this transcriptional synergy by using SUMOylation deficient PR mutants and PR specifically deSUMOylated by Sentrin-specific proteases (SENPS). We show that deSUMOylation of a small pool of receptors by catalytically competent SENPS globally modulates the cooperativitydriven transcriptional synergy between PR observed on exogenous promoters containing at least two progesterone-response elements $\left(\mathrm{PRE}_{2}\right)$. This occurs in part by raising PR sensitivity to ligands. The C-terminal ligand binding domain of PR is required for the transcriptional stimulatory effects of N-terminal deSUMOylation, but neither a functional PR dimerization interface, nor a DNA binding domain exhibiting PR specificity, are required.

Conclusion: We conclude that direct and reversible SUMOylation of a minor PR protein subpopulation tightly controls the overall transcriptional activity of the receptors at complex synthetic promoters. Transcriptional synergism controlled by SENP-dependent PR deSUMOylation is dissociable from MAPK-catalyzed receptor phosphorylation, from SRC-1 coactivation and from recruitment of histone deacetylases to promoters. This will provide more information for targeting PR as a part of hormonal therapy of breast cancer. Taken together, these data demonstrate that the SUMOylation/deSUMOylation pathway is an interesting target for therapeutic treatment of breast cancer.
\end{abstract}

\section{Background}

Progesterone plays a key role in the development, differentiation and maintenance of normal and malignant female tissues. Its effects are mediated by progesterone receptors (PRs), members of the steroid hormone receptor superfamily of ligand-dependent transcription factors. PRs exist as two major, functionally different [1] isoforms-PR-A $(\sim 94 \mathrm{kDa})$ and PR-B $(\sim 110 \mathrm{kDa})$. They are multidomain proteins consisting of a central DNA-binding domain (DBD); large $\mathrm{N}$-termini with a proximal activation function (AF-1) common to both isoforms; a distal AF-3 in the B-upstream segment (BUS) restricted to PR-B; and at their C-termini, a nuclear localization signal in a hinge region upstream of an AF-2-containing ligand binding domain

\footnotetext{
* Correspondence: hany.abdel-hafiz@ucdenver.edu

'Division of Endocrinology, Department of Medicine, Anschutz Medical

Campus, University of Colorado, Aurora, CO 80045, USA

Full list of author information is available at the end of the article
}

(LBD) [1-5]. PRs are transactivators that can be tethered to DNA through other transcription factors [6-10] but more commonly are bound directly to DNA at palindromic progesterone-response elements (PREs) [11]. The two isoforms bind DNA with equivalent affinity [12] so this cannot explain their functional differences. Rather, dissimilar coregulator recruitment has been invoked for their differences [13]. These coactivators or corepressors facilitate receptor/DNA occupancy, chromatin remodeling and recruitment of general transcription factors associated with the RNA polymerase II holocomplex [14]. Function of the receptors and their coregulators are in turn controlled by posttranslational modifications including phosphorylation, acetylation, ubiquitination and SUMOylation [15] that influence hormone sensitivity and promoter selectivity, among others [16]. Ubiquitination for example, promotes ligand-dependent PR protein downregulation via proteasomal degradation, which paradoxically 
maximizes transcriptional activity [17]. Because these modifications are reversible, enzymes that dephosphorylate, deacetylate, deubiquitinate and deSUMOylate PRs also alter activity $[16,18-20]$, so that permutations of these modifications undoubtedly play a large role in the complex signaling patterns ascribed to the receptors [1].

\section{Transcriptional synergy and PR SUMOylation}

Additional complexity arises from the structure of DNA to which PRs bind. Cooperativity among receptors bound at compound promoters consisting of two or more PREs results in synergism defined as a "more-than-additive" transcriptional effect [21]. Iniguez-Lluhi and Pearce [21] first identified a short synergy control (SC) motif in glucocorticoid receptors (GR) that disrupted synergy on promoters with multiple response elements. Its mutation induced strong synergistic effects but only at compound response elements. The $\mathrm{SC}$ motif turned out to be a SUMOylation site at which conjugation of SUMO-1, a 97 amino acid (aa) Small Ubiquitin-like Modifier, disrupted synergy [22-24]. Similar sites in both GR and PR [15] contain a lysine (Lys, $\mathrm{K}$ ) residue embedded in the consensus sequence $\Psi \mathrm{KxE}$ (where $\Psi$ is a large hydrophobic amino acid, and $x$ is any amino acid) located in the $\mathrm{N}$-terminal AF-1 domains of the receptors. For human $\mathrm{PR}-\mathrm{B}$ this sequence is centered at $\mathrm{K} 388$, and at a homologous site of PR-A. Monomeric SUMO-1 covalently binds this site through a series of dynamic and reversible enzymatic reactions involving an E1 SUMO activating enzyme, an E2 conjugating enzyme (Ubc9) and E3 ligases (PIASs; Protein Inhibitors of Activated STAT (Signal Transducer and activator of transcription)). DeSUMOylation is catalyzed by one of six human Sentrin-specific proteases (SENPs) that target SUMO. Largely due to their roles in modifying the activity of steroid receptors, both Ubc9 and PIAS have at times been classified as transcriptional coregulators [25-27]. Mouse knockouts of Ubc9 or SENP1 are embryonic lethal, demonstrating that the balance of SUMOylation and deSUMOylation is essential for development $[28,29]$. Most, but not all steroid receptors-the exception appearing to be estrogen receptors (ER)-are targets of SUMOylation. This is consistent with the fact that phylogenetic and sequence alignments of GR, mineralocorticoid receptors (MR), androgen receptors (AR) and PR links them to a steroid receptor subfamily characterized by much larger $\mathrm{N}$-termini (ranging from 420 to 602 aa) than the N-termini of ER $\alpha$ or $\operatorname{ER} \beta$ (184 and 148 aa, respectively). As a result in vitro translated $\mathrm{AR}$ and $\mathrm{GR}$, but not $\mathrm{ER} \alpha$ or $\mathrm{ER} \beta$, are SUMOylated [30].

SUMO conjugation of PR-B at K388 (or the PR-A equivalent) is hormone dependent and occurs via PIAS1 [31] or PIAS3 [32]. This suppresses PR -dependent transcription of promoters containing multiple PREs but not a single PRE [6,18,33-35]. Additionally, overexpression of PIAS3 can induce PR-B SUMOylation at K7 and K531 [32] but the physiological relevance of this is unclear. SUMO is deconjugated from the receptors by SENPs, which, like deSUMOylation by mutation of K388, dramatically enhances PR transcriptional activity [18]. The relationship between the transcriptional efficacy of deSUMOylation and the role of ligand-dependent PR downregulation are contradictory. Zhang and coworker [36] showed that mutation of PR-B at K388 retards progesterone-induced degradation through the ubiquitin-proteasome pathway. In contrast, we and others $[6,18]$ have shown that PR K388R mutants undergo accelerated ligand-dependent downregulation thereby explaining their heightened transcriptional activity.

In the present study we analyze the functional effects of SENP-induced PR deSUMOylation in detail. Our results indicate that on a compound promoter, SENP1 enhances transcription in a dose-dependent manner, but this requires full-length PR. However enhanced transcription is independent of PR DNA binding specificity or the PR S294 phosphorylation site. By deSUMOylating PR, SENP increases PR sensitivity to hormone. The histone deacetylase inhibitor Trichostatin A (TSA) has a marked biphasic effect. At high concentrations, which promote global histone hyperacetylation and modify many proteins [37], TSA strongly suppresses transcription and this is reversed by the coactivator SRC-1. However, low TSA concentrations upregulate PR-dependent transcription. This effect of TSA is uncoupled from inhibition by SUMOylation indicating that HDAC activity is not involved in transcriptional synergy controlled by SENP1.

\section{Results}

\section{SENP and PR deSUMOylation}

\section{SUMOylation and the promoter context of $P R$}

\section{transcriptional synergy}

Figure $1 \mathrm{~A}$ is a schematic of PR-B and PR-A showing location of the single $\psi \mathrm{KxE}$ SUMO-conjugation motif centered at K388 of PR-B (and a homologous K224 of PR-A). Also shown are 3 hormone dependent serine (S102, S294 and S345) and multiple other (thin lines) $\mathrm{N}$-terminal phosphorylation sites, and a hinge domain KxKK (aa 638-641) acetylation site.

We previously showed [6] that SUMOylation at K388 (or K224) is hormone-dependent and suppresses PR-B and PR-A-regulated transcription of an exogenous promoter containing two $\left(\mathrm{PRE}_{2}\right)$ or more palindromic PREs but not a single PRE. To assess the generality of this, we used the MMTV-LTR, which contains 1 palindromic PRE and 3 PRE half-sites. In contrast to GRs that prefer the palindrome, the half-sites are preferentially used by PRs [38], possibly as monomers [39]. To examine the role of PR SUMOylation on transcriptional synergy involving PRE 

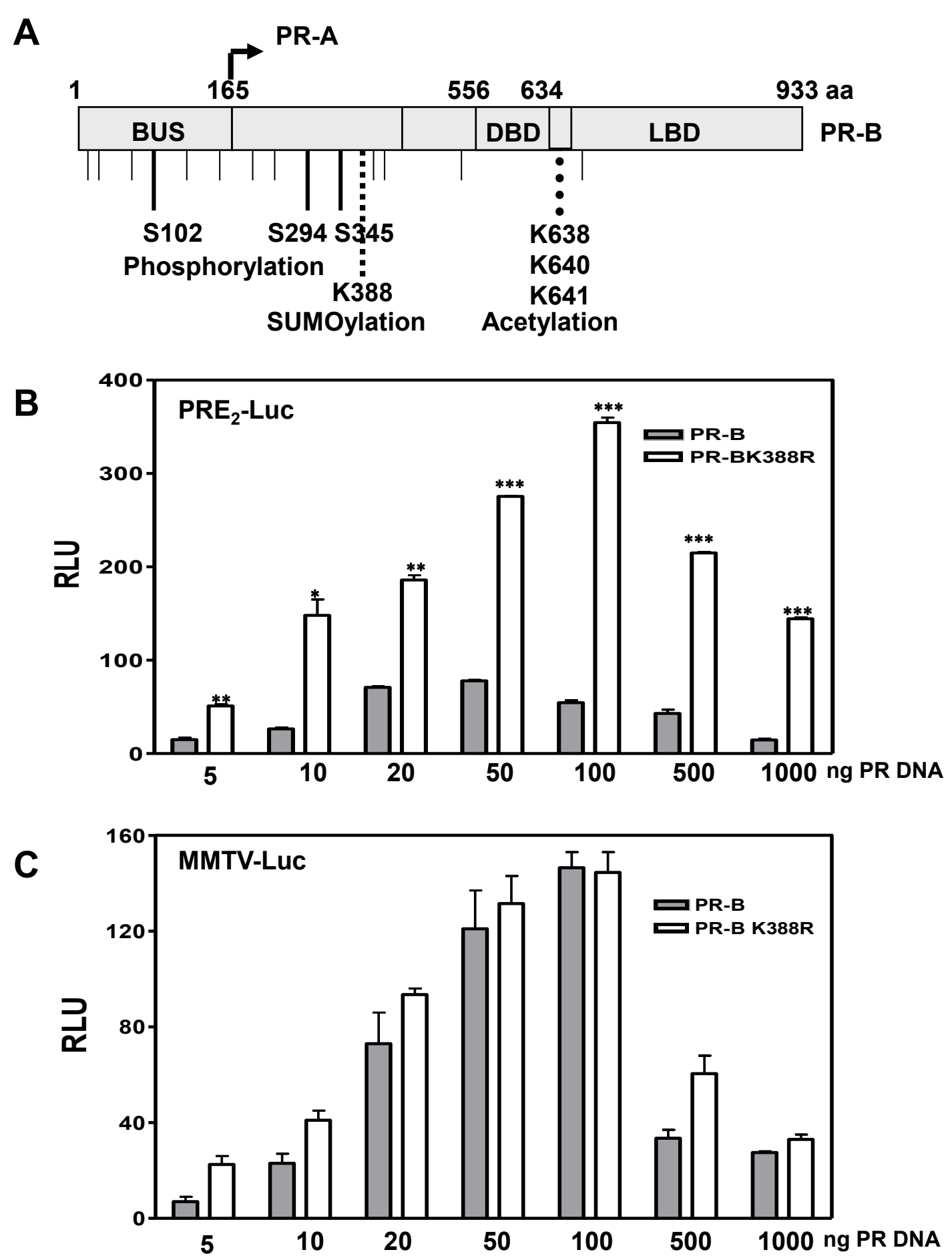

Figure 1 Modulation of PR transcriptional activity by SUMO-1 depends on the promoter context. A) schematic of PR-A and PR-B showing the location of hormone dependent phosphorylation sites; the Lys-388 SUMO conjugation site within an IKEE motif; and an acetylation consensus KxKK site (amino acids 638-641). BUS, B-upstream segment; DBD, DNA binding domain; LBD, ligand binding domain. B) HeLa cells were transfected with $2 \mu \mathrm{g}$ of PRE2-Luc reporter, or C) MMTV-Luc, together with 5-1000 ng of wild-type PR-B or mutant PR-B K388R expression vectors and Renilla-Luc as an internal control. The cells were treated 24 hrs with $10 \mathrm{nM}$ R5020, then harvested and lysed. The extracts were assayed for luciferase activities. Luciferase activity is expressed in relative light units (RLU). Data represent triplicates $( \pm$ SD). Statistical significance was computed by unpaired student's $t$ test. ${ }^{*} p<0.05$. 
half-sites, HeLa cells were transfected with 5-1000 ng of DNA encoding wild-type PR-B or the SUMOylation-deficient K388R PR-B mutant [33], together with the $\mathrm{PRE}_{2}$ Luc (Figure 1B) or MMTV-Luc (Figure 1C) reporters, in the presence of the progestin R5020. PR-B were tested since they are more potent transactivators of the MMTVLTR than PR-A [40]. On $\mathrm{PRE}_{2}$-Luc, wild-type PR-B were transcriptionally active, and mutation of their K388 SUMOylation motif synergistically raised transcription further as receptor concentrations were increased between 5 and 100 ng DNA. High PR concentrations (500 and 1000 ng DNA) led to a decrement in transcription likely due to transcription factor "squelching" [33]. Wild-type PR-B dependent transcription on MMTV-LTR showed a similar dose-dependent increase. However, absolutely no transcriptional synergy was observed with the K388R PR-B mutant suggesting that SUMOylation does not control synergy on PRE half-sites. Most of the studies below use $\mathrm{PRE}_{2}$-Luc

\section{DeSUMOylation by SENP}

The K388R PR mutant is an artificial construct while proteins are naturally deSUMOylated by SENPs in vivo [18]. To examine effects of in vivo PR deSUMOylation, wild-type PR-B (1 $\mu \mathrm{g}$ DNA) and GFP-SUMO1 (0.1 $\mu \mathrm{g}$ DNA) were cotransfected into HeLa cells together with SENP1 or SENP2 expression vectors $(0.1 \mu \mathrm{g}$ DNA), and unliganded or liganded PR-B SUMOylation states were assessed by immunoblotting (Figure 2A). PR-B are not SUMOylated by ligand in the absence of SUMO-1 (lane 2 ), or by SUMO-1 in the absence of ligand (lane 3), but approximately $5 \%$ of the receptors are SUMOylated when both are present (lane 4). However, in cells coexpressing SENP1 (lane 6) or SENP2 (lane 8) SUMO1PR conjugates are essentially absent. A R630L, K631M SENP1 mutant (SENP1m), whose catalytic function is disabled [20], was unable to deSUMOylate PR (Additional file 1: Figure S1A).

We next tested effects of increasing concentrations of DNA (20-1000 ng) encoding SENP1, SENP1m and SENP2 on $\mathrm{PRE}_{2}$-Luc transcription by R5020-liganded, wild-type PR-B transiently expressed in HeLa cells (Figure 2B) or stably expressed in T47D breast cancer cells (Additional file 1: Figure S1B). Analogous to the K388R SUMOylation-deficient PR-B mutant, deSUMOylation by SENP1 and SENP2 strongly enhanced the transcriptional activity of wild-type liganded PR-B in both cell types in a dosedependent manner. The SENP1m control was ineffective (Figure 2B). It is of interest that these extensive transcriptional effects of SUMOylation/deSUMOylation are regulated by a minor subpopulation of PR molecules (Figure 2A). Indeed, the PR SUMOylation state and its control of transcription applies even to weak progestin agonists as shown by the fact that deSUMOylation by SENPs intensifies transcription by the mixed agonist/ antagonist RU486 [41], but has no effect on transcription by the pure antagonist ZK98299 (Additional file 1: Figure S1C

The above results indicate that the activity of agonistoccupied PRs can be regulated dynamically and reversibly by SUMOylation/deSUMOylation of a small receptor subpopulation. To demonstrate whether this is a direct effect on PRs, or an indirect effect on SUMOylation of coregulators brought to the transcription complex by PRs, wt PR-B (Figure 3A, C) or the PR-B K388R mutant (Figure 3B, D) were co-expressed with increasing (20-1000 ng) concentrations of SENP1, and tested on $\mathrm{PRE}_{2}$-Luc (Figure 3A, B) or MMTV-Luc (Figure 3C, D). SENP1 enhanced PR-B-dependent transcription in a dose-dependent manner on $\mathrm{PRE}_{2}$ Luc, but was ineffective in modifying transcription by PR-B $\mathrm{K} 388 \mathrm{R}$ on the same reporter, indicating that the response to SENP1 requires the PR SUMOylation site. This was confirmed on MMTV-Luc (or a single PRE, not shown) where SENP1 had no effect despite strong transcription with wildtype PR-B, confirming that the PREs of MMTV-LTR are not PR SUMOylation sensitive (Figure 1). We conclude that SENP1 modifies PR-dependent transcription directly at the PR SUMOylation site, which is also required for the cooperativity-driven synergy observed on a $\mathrm{PRE}_{2}$.

\section{SENP action on PR: Mechanisms Activation functions}

To assess whether SENP modifies activity via AFs, two PR deletion mutants were tested: 1) NT-B, a constitutively active PR N-terminal construct [42] containing AF$3, \mathrm{AF}-1$ and its $\psi \mathrm{KxE}$ SUMOylation site, linked to the DBD but missing the C-terminal AF-2 of the LBD (Figure 4A); 2) DBD-LBD, the PR DBD linked to the Cterminal LBD and its AF-2 (Figure 4B). The constructs (100 ng DNA) were transfected into HeLa cells expressing increasing concentrations (20-1000 ng) of DNA encoding SENP1 or SENP1m and transcription was measured using $\mathrm{PRE}_{2}$-Luc. NT-B is strongly active in the absence of ligand (Figure 4A). Despite containing the PR SUMOylation site, SENP1 was unable to further increase this strong constitutive activity. This confirms that NT-B is not SUMOylated in the absence of the LBD [33], making it insensitive to SENP1. Rather, we observe a dosedependent repression by SENP1 requiring its catalytic activity (compare SENP1 vs. SENP1m) suggesting an effect by SENP1 on deSUMOylation of N-terminal interacting coregulatory factors. Wild-type SENP1 does not have a repressive effect on the weak ligand-dependent transcription of DBD-LBD (Figure 4B); likely the target of different, possibly non-SUMOylated, C-terminal interacting coregulators.

\section{DNA binding specificity}

Next we assessed the role of the PR DBD in mediating effects of SENP1 using two additional constructs: 1) a 


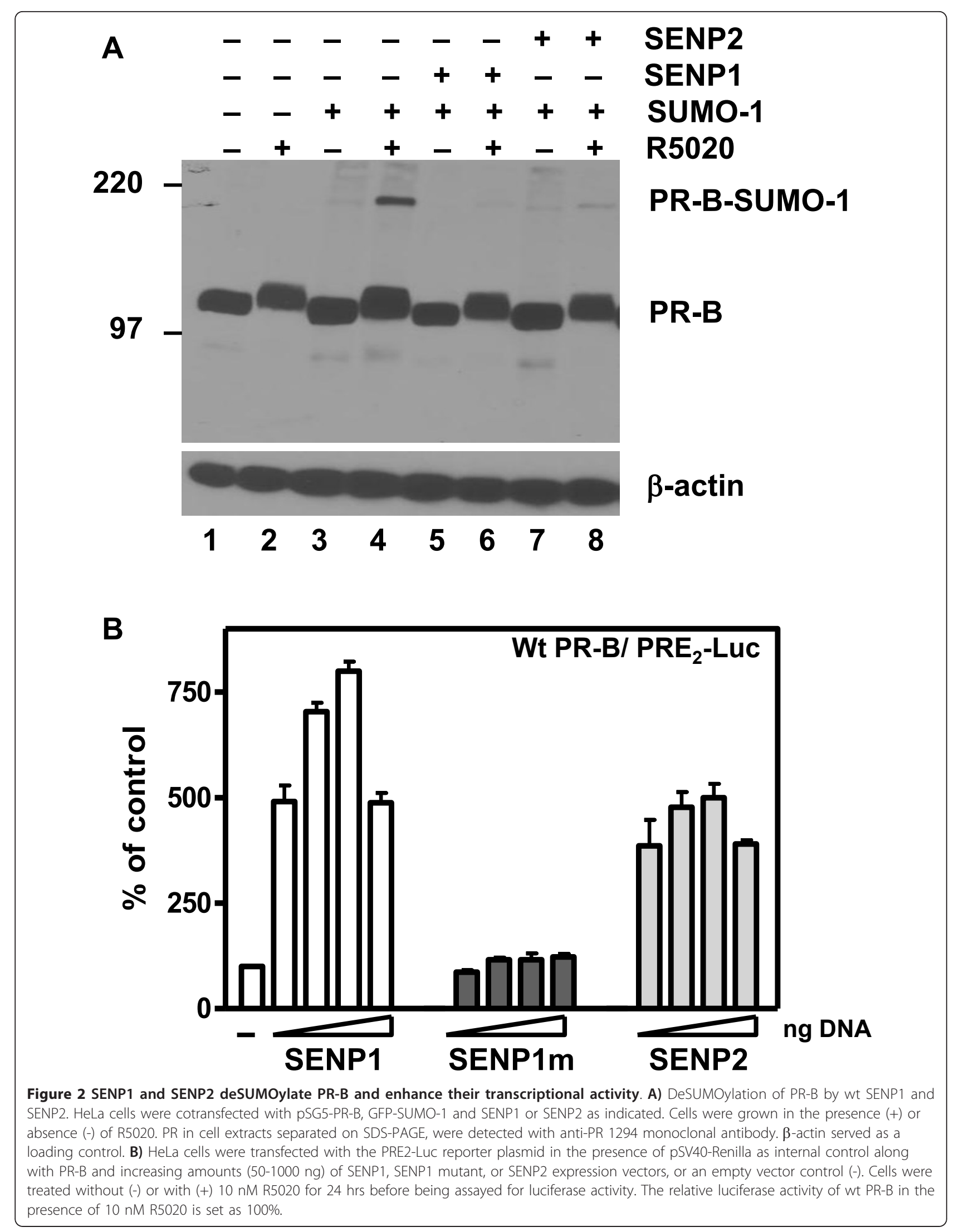




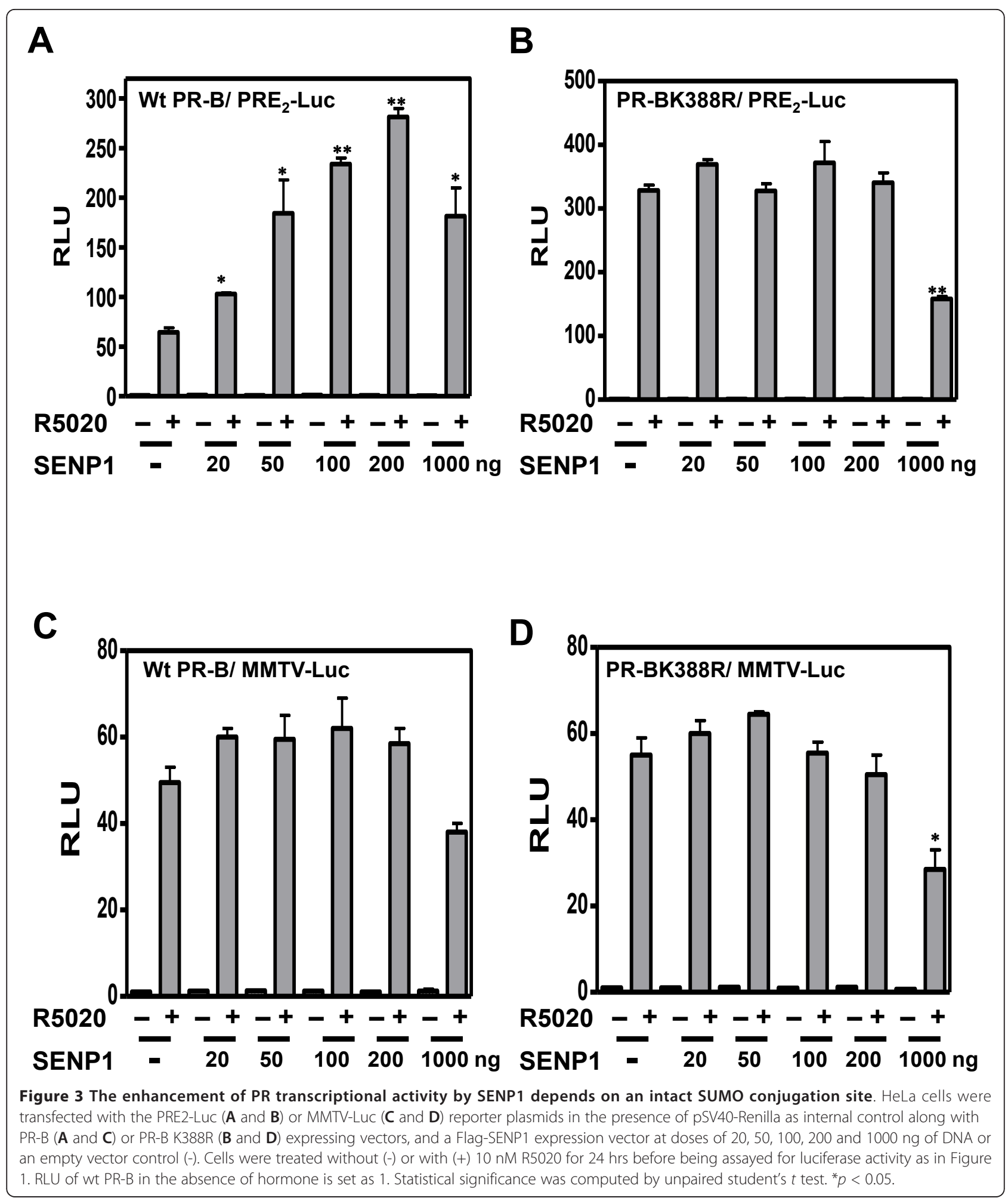

full-length PR-B Spec "specificity" mutant in which the PR DBD was replaced by the DBD of ER (Figure 4C), and 2) wild-type ER (Figure 4D). Both were tested on tandem estrogen response elements $\left(\mathrm{ERE}_{2}\right)$ linked to luciferase. The PR-B specificity mutant was treated with R5020 (since it contains a PR LBD); ER was treated with $17 \beta$-estradiol. The receptor-encoding constructs (100 ng DNA) were transfected into HeLa cells without or with 


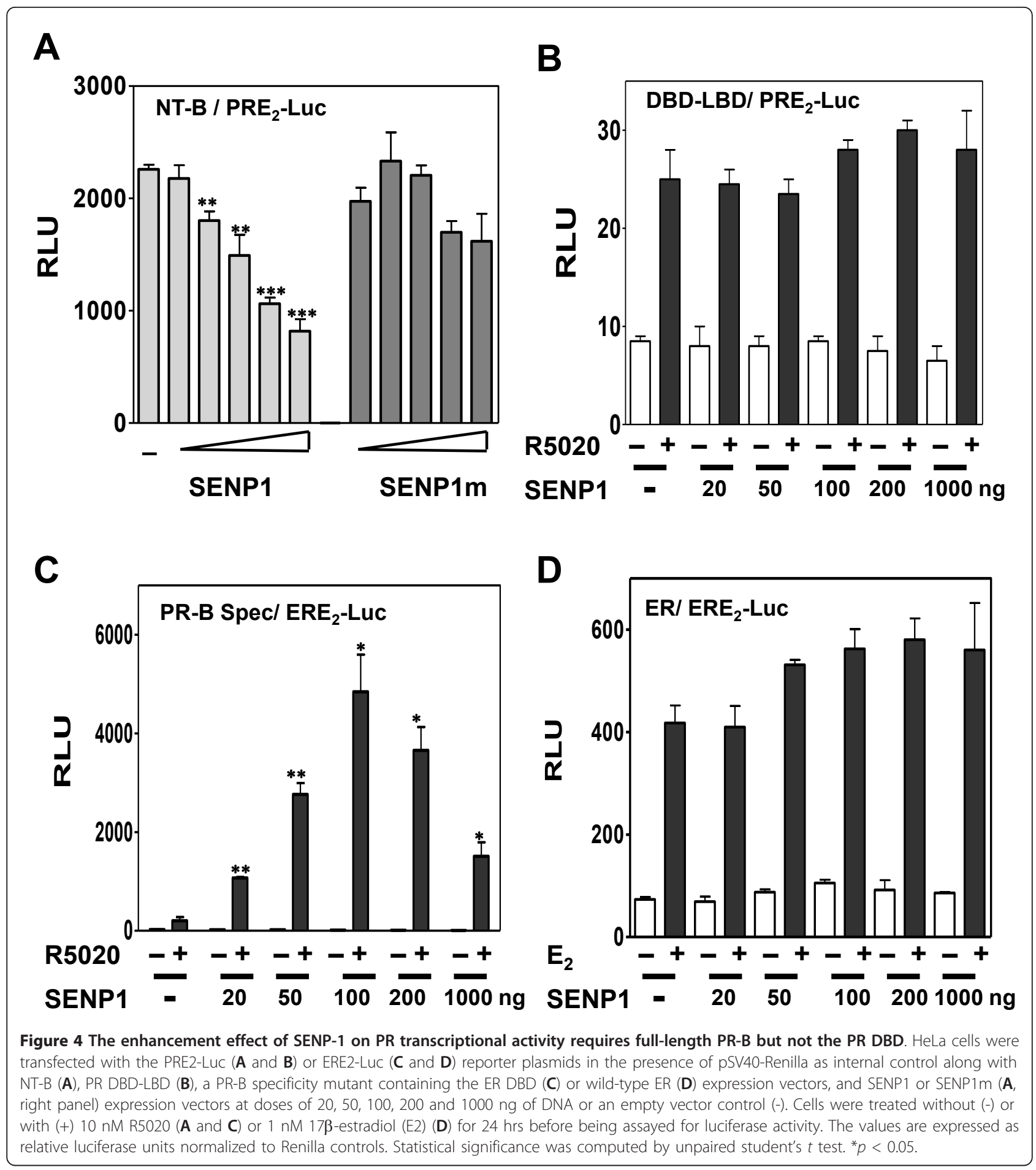

hormones together with increasing (20-1000 ng) SENP1 concentrations. The PR-B specificity mutant exhibited weak ligand-dependent transcriptional activity, which was dramatically enhanced by SENP1-mediated deSUMOylation in a dose-dependent manner. This suggests that unlike the PR LBD, neither the PR DBD nor its DNA binding site influence SUMOylation of the PR N- terminus. The DBD dimer interface of steroid receptors stabilizes binding to palindromic HREs. Interestingly, disruption of the dimer interface markedly increases transcriptional activity of receptors bound to multiple PREs (Additional file 1: Figure S2) indicating that DBD dimerization generally suppresses synergy. Wild-type ERs were unaffected by SENP1, consistent with our 
previous report [33] that ERs are not substrates of SUMOylation. This failure is not controlled by the ER DBD or EREs since both support SUMOylation in the context of PR-B. Unlike N-terminal coregulatory proteins of PR (Figure 4A), ER transcriptional coregulators appear to be unaffected by their SUMOylation state.

\section{Sensitivity to ligand}

Since SUMOylation reduces PR-B sensitivity to hormone $[6,18]$ we speculated that deSUMOylation by SENP would reverse this effect. To test this, HeLa cells expressing constant levels of PR-B or the PRB K388R mutant (50 ng DNA), in the absence or presence of constant SENP1 levels (100 ng DNA) were treated $24 \mathrm{hrs}$ with R5020 at doses ranging from $10^{-15}$ to $10^{-8} \mathrm{M}$. Transcription levels on $\mathrm{PRE}_{2}$-Luc were plotted as a percent of maximal induction by $10^{-8}$ M R5020 above "no hormone" controls. Curve fitting was performed by Prism Graph as described under "Experimental Procedures" (Figure 5). SENP1 reduced the dose of R5020 required for half-maximal transcription (EC50) by wild-type PR-B $\sim 4.7$-fold, from $2.74^{-11} \mathrm{M}$ to $5.85^{-12} \mathrm{M}$ (Figure $5 \mathrm{~A}$ ). SENP1 had little or no effect on the EC50 $\left(\sim 1.5^{-11} \mathrm{M}\right)$ of the SUMOylation deficient K388R mutant whose intrinsic R5020-binding affinity exceeded that of wildtype PR $\sim 2$-fold. This indicates that deSUMOylated PR are exquisitely sensitive to very low hormone concentrations; also explaining enhancement of the agonist properties of RU486 (Additional file 1: Figure S1C). Saturating hormone concentrations were similar for the two receptors (Figure 5B, D).

\section{SENP, PR phosphorylation and MAPK signaling}

PRs are phosphorylated on multiple serine residues (Figure 1), three of which-S102, S294 and S345-are currently known to be ligand-dependent [43-45]. Contradictory reports indicate on the one hand that PR-B phosphorylation is uncoupled from SUMOylation [6], and on the other that MAPK-catalyzed S294 phosphorylation antagonizes PR-B SUMOylation [18]. To assess interactions between deSUMOylation and MAPK signaling, we analyzed transcription in the presence of SENP1 (100 ng) and MAP/ERK Kinase Kinase (MEKK1; $100 \mathrm{ng}$ ), a strong activator of MAPK-dependent PR phosphorylation, using wild type PR-B, PR-B S294/345 phosphorylation-deficient mutants, or PR-B K388R SUMOylation-deficient mutants (Figure 6). On wild-type PR (Figure 6A), SENP1 and MEKK1 increased transcription equally, and their combined effects were additive. A key difference between the two is that SENP1 does not alter basal transcriptional activity, but MEKK raises it (Figure 6A, inset). The phosphorylation deficient mutant (Figure 6B) remained responsive to SENP1, dissociating S294/345 phosphorylation from deSUMOylation. Interestingly, MEKK1 also activated this mutant suggesting either that other PR sites, or PR coregulatory proteins, are MEKK-regulated in the S294/345-deficient receptors. Finally, SENP1 failed to hyperactivate the constitutively active K388R mutant (Figure 6C), as would be expected. However, MEKK1 was able to activate this SUMOylation-deficient PR or the constitutively active NT-B (Additional file 1: Figure S3A), uncoupling MEKKdependent activation from PR K388 SUMOylation. Activation of MAPK signaling by overexpressing MEKK1 has complex, concentration-dependent effects on PR SUMOylation. At low concentrations, MEKK1 induces ligand-independent PR SUMOylation (Additional file 1: Figure S3B, lanes 3). At high concentrations, MEKK1 suppresses hormone-dependent PR SUMOylation (Additional file 1: Figure S3B, lanes 6\&8).

SENP, histone deacetylases (HDAC) and SRC-1 coactivation Repression of the Elk-1 transcription factor by SUMOylation couples with recruitment to promoters of histone deacetylases, to further repress Elk-1 target genes [46]. This suggests that HDACs are involved in the transcriptional repression by SUMO [46]. We asked whether HDACs are involved in the synergy control and regulation of PR activity by SENP1. We first analyzed baseline effects of trichostatin A (TSA)-an HDAC inhibitor that brings about chromatin decondensation [47]-on PR-B-dependent transcription of $\mathrm{PRE}_{2}$-Luc (Figure 7A). There was a marked biphasic response. Compared to untreated controls, low doses of TSA (50 and $100 \mathrm{nM}$ ) upregulated both basal and liganded PR-B dependent transcription, while excessive TSA doses (500 and $1000 \mathrm{nM}$ ) were strongly inhibitory. Similar inhibitory effects of TSA have been attributed to incompatibility between hyperacetylation of chromatin and assembly of coactivators on the RNA pol II complex [48]. To assess this, we analyzed the ability of steroid receptor coactivator 1 (SRC-1) to coactivate PR-B on $\mathrm{PRE}_{2}$-Luc, at low (100 nM) or high (500 nM) TSA concentrations. At low TSA concentrations (Figure 7B), HeLa cells express sufficient endogenous SRC-1 to maximally coactivate PR-B dependent transcription, and exogenous addition of excess SRC-1 (20-1000 ng) does not alter these already high levels. However, high TSA concentrations (Figure 7C) repress transcription controlled by endogenous coactivators more than 90\%, which exogenous SRC-1 (20-1000 ng) is able to reverse. These data support the conclusion that in HeLa cells, promoter hyperacetylation suppresses coactivator recruitment to DNA-bound PR. Additionally, we noted that high concentrations of TSA stabilize PR-B protein levels (Figure 8A; compare lane 1 vs. lanes 2-5), and prevent ligand-dependent PR-B downregulation (compare lanes 6,7 vs. 8-10). Suppression of unliganded and/or liganded PR protein turnover would also impede transcription [49].

The relationship between HDAC inhibition and PR deSUMOylation was therefore probed using low (100 nM) TSA concentrations together with the deSUMOylase 


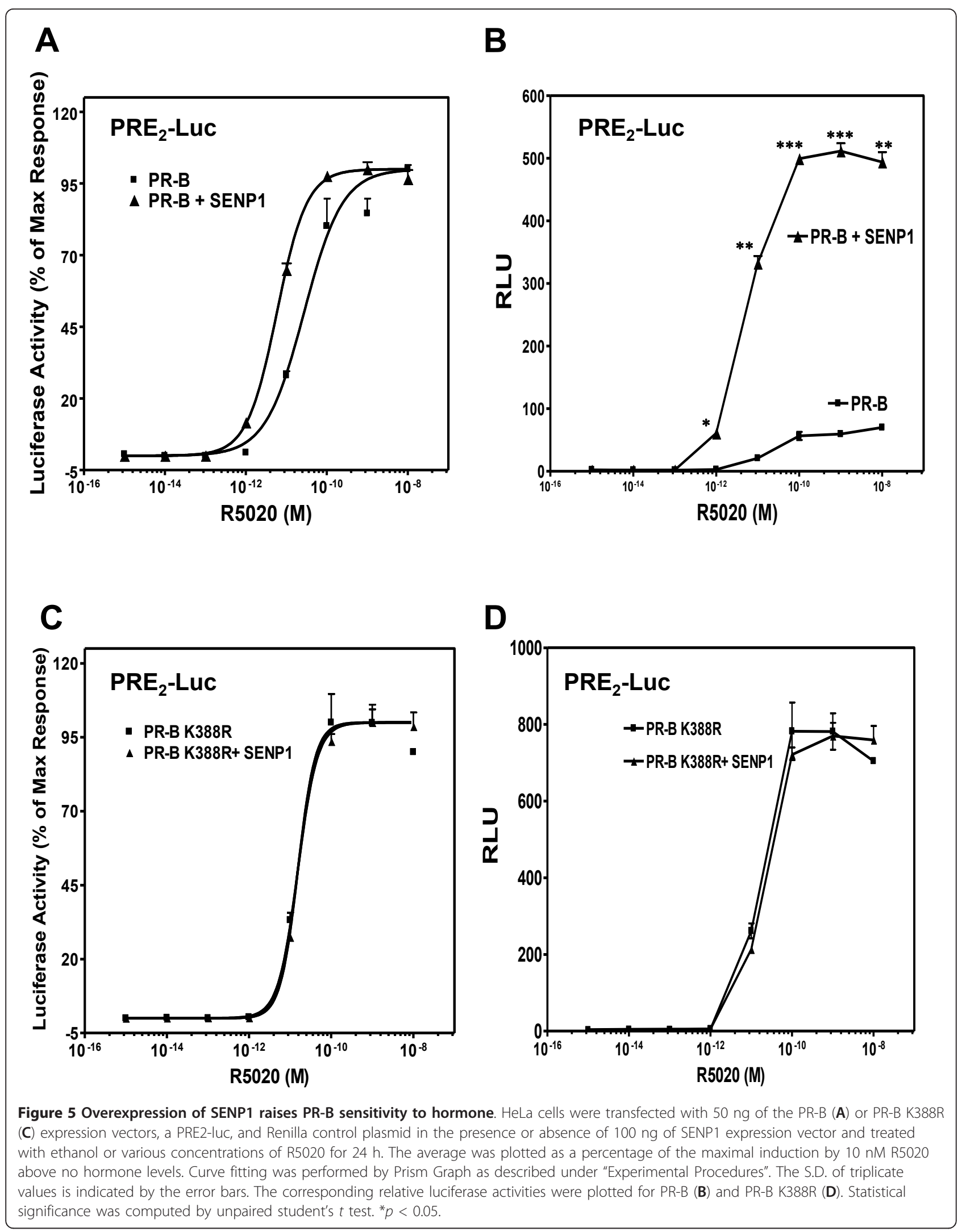




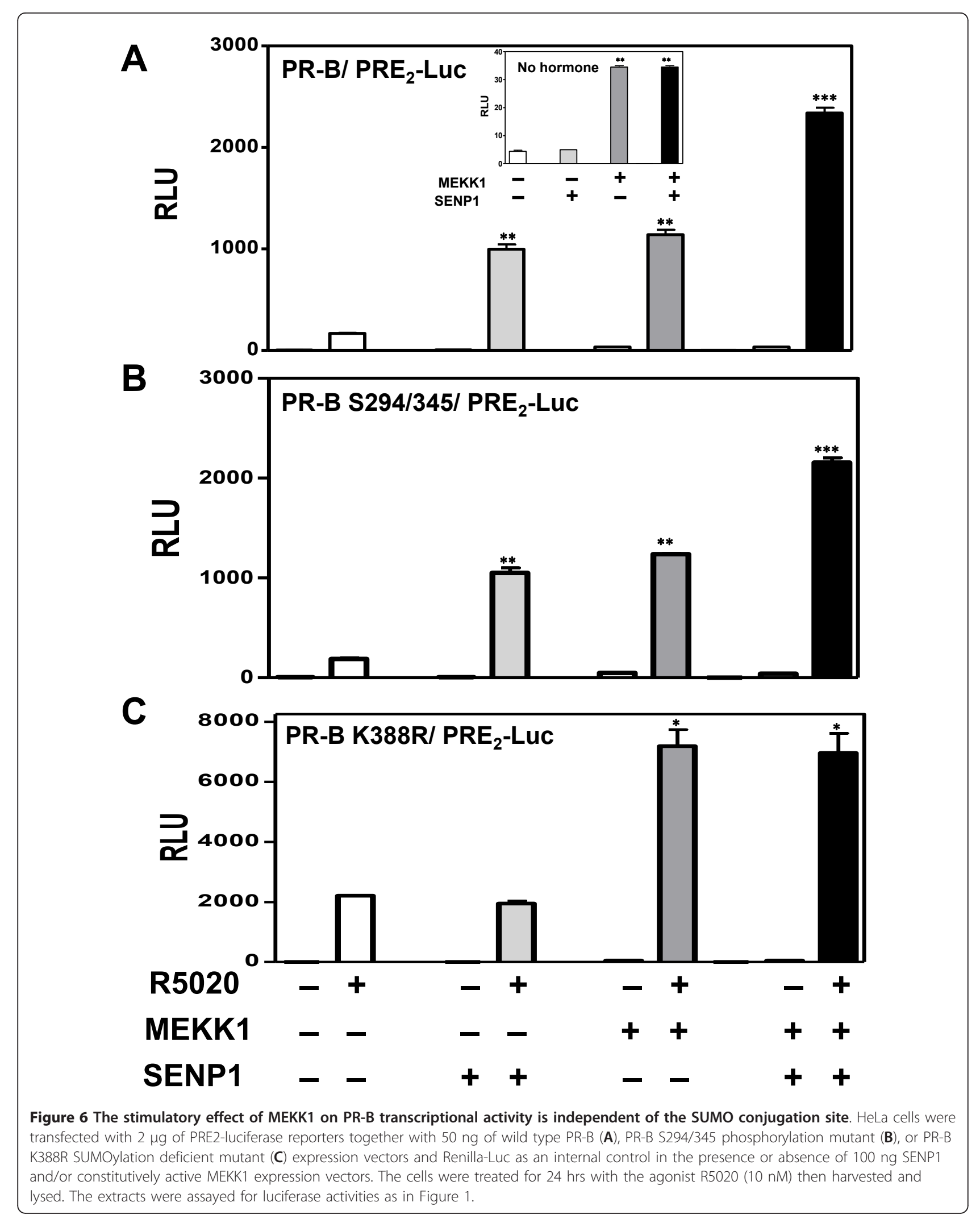



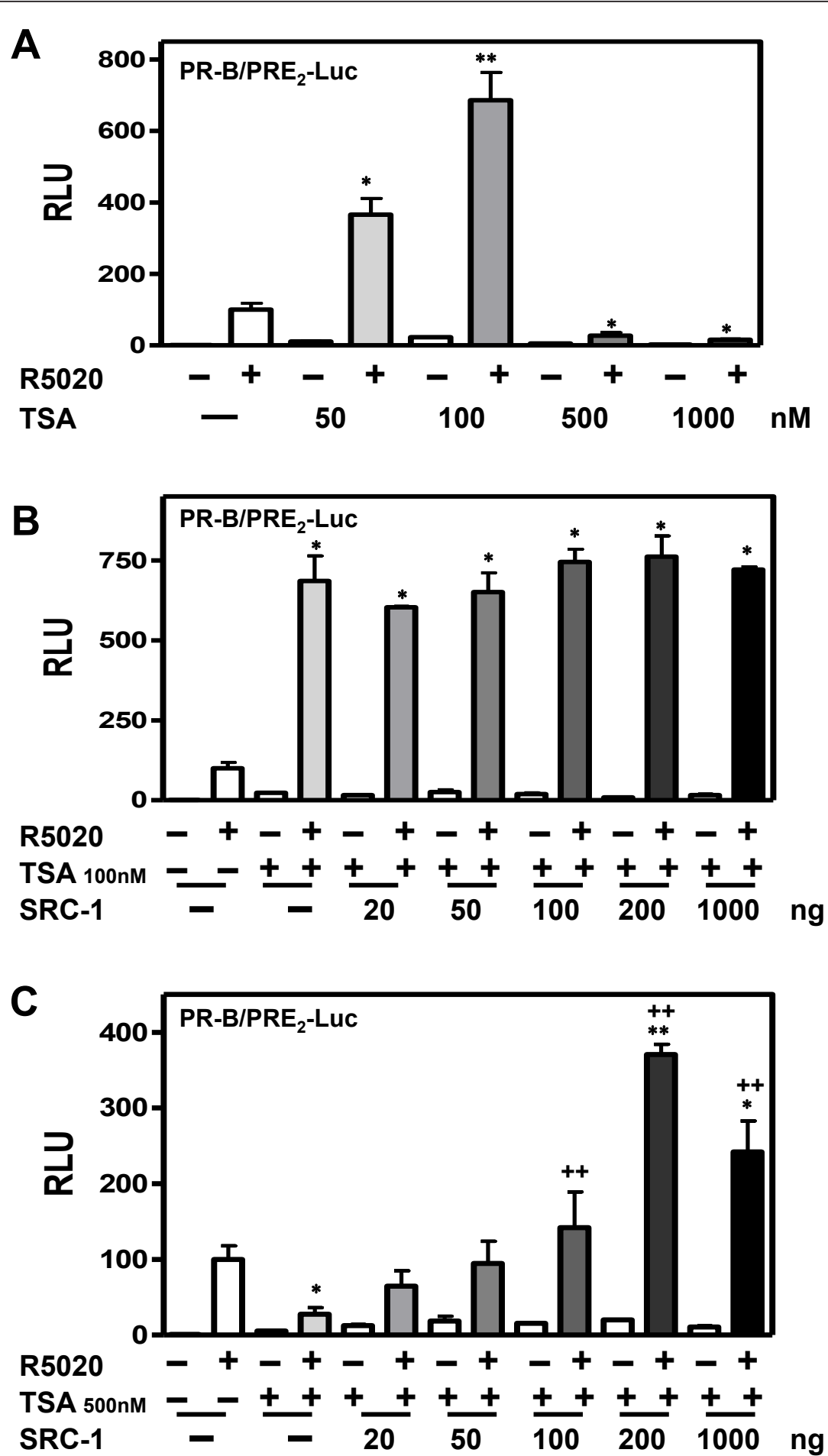

Figure 7 SRC-1 reverses the inhibitory effect of the HDAC inhibitor TSA on the PR transcriptional activity. A) HeLa cells were transfected with $2 \mu \mathrm{g}$ of PRE2-luciferase reporters together with $50 \mathrm{ng}$ of a PR-B and Renilla-Luc as an internal control. The cells were treated for 24 hrs with

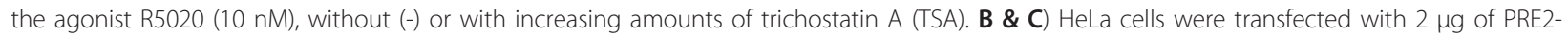
luciferase reporters together with $50 \mathrm{ng}$ of a PR-B expression vector and Renilla-Luc as an internal control in the absence or the presence (+) of increasing amount of SRC1. The cells were treated for 24 hrs with the agonist R5020 (10 nM), without (-) or with (+) 100 nM (B) or 500 nM (C) of TSA then harvested and lysed. The extracts were assayed for luciferase activities as in Figure 1. (*) Compared with control and (+) compared with TSA treatment. 


\section{TSA}

PR-B

\section{$\beta$-actin}

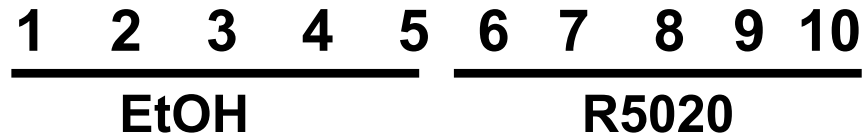

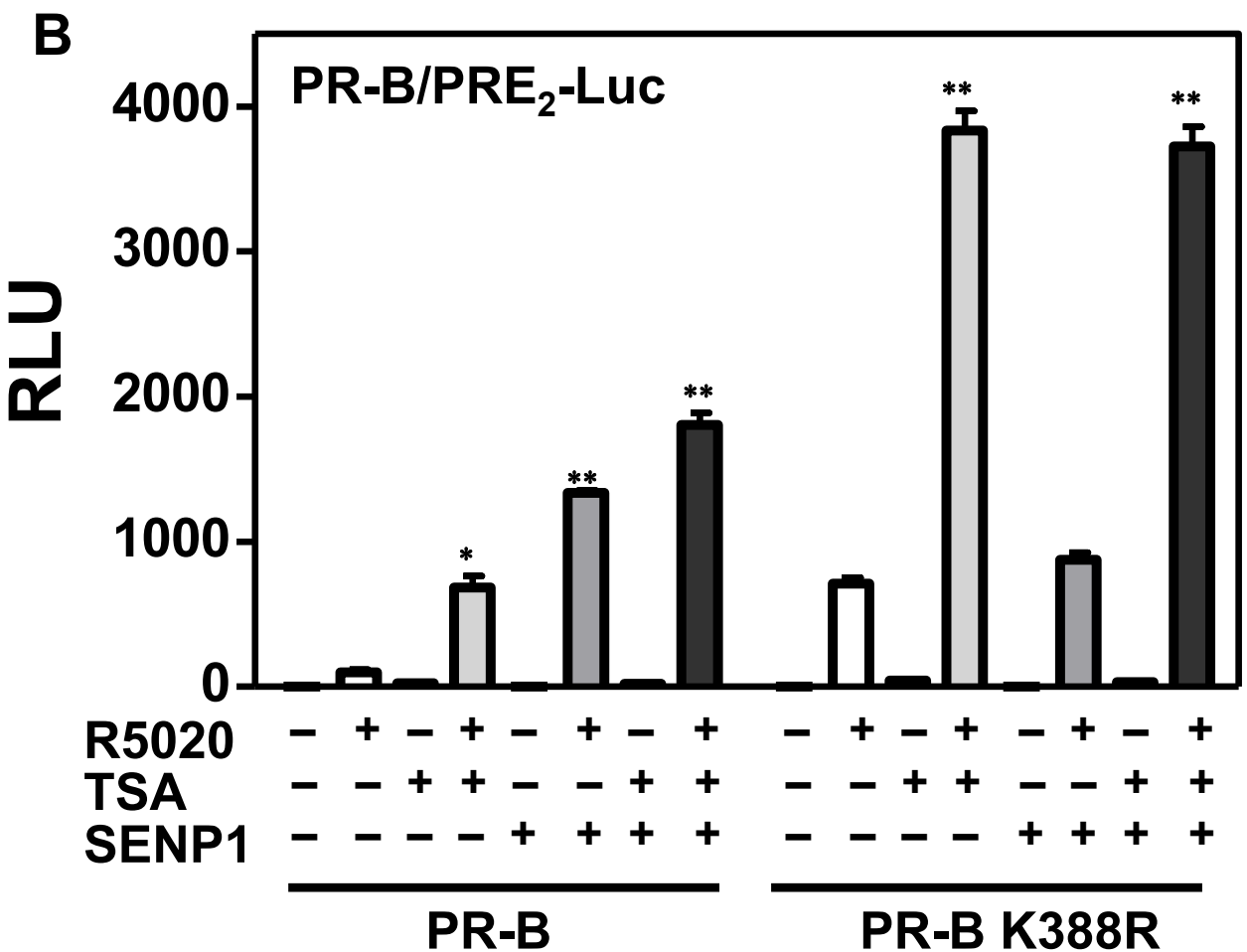

Figure 8 HDACs are not a major target for SENP1 action on PR transcriptional activity. A) TSA enhances PR-B protein stability. HeLa cells were transiently transfected with expression vectors encoding wild type PR-B. Cells were treated 24 hrs without (-) or with (+) 10 nM R5020 in the presence of increasing concentration of TSA. Western blot analysis was performed on cell extracts probed with the anti-PR1294 monoclonal and anti $\beta$-actin antibodies. B) HeLa cells were transfected with $2 \mu \mathrm{g}$ of PRE2-luciferase reporters together with $50 \mathrm{ng}$ of a PR-B (left), or the PR-B K388R mutant (right) expression vectors and Renilla-Luc as an internal control in the presence or absence of 100 ng SENP1 expression vectors. The cells were treated for 24 hrs with the agonist R5020 (10 nM), without (-) or with (+) 100 nM TSA then harvested and lysed. The extracts were assayed for luciferase activities as in Figure 1. Statistical significance was computed by unpaired student's $t$ test. ${ }^{*} p<0.05$. 
SENP1. HeLa cells were transfected with wild-type PR-B (Figure 8B, left) or the PRB K388R mutant (Figure 8B, right) in the absence (-) or presence (+) of SENP1 (100 ng) and/or TSA (100 nM). On wild-type PR-B, either TSA alone or SENP1 alone caused the expected increase in transcription. The two together were additive, suggesting a lack of interaction between them. On the K388R SUMOylation-deficient mutant, TSA was especially potent in hyperactivating the already strong basal activity. SENP1, as expected, had no effect on this basal activity. When combined with TSA, SENP1 also had no effect, suggesting that HDAC activity does not markedly contribute to transcription synergy.

\section{Discussion}

SUMO-dependent transcriptional repression and synergy Various regulators of SUMO-dependent transcriptional repression have been proposed, which include chromatinassociated proteins [50], histone deacetylases [46], the SUMO-binding death-domain associated protein DAXX [51], the DEAD-box protein DP-103 [52], and the nuclear matrix protein NXP-2 [53]. The link between relief from SUMOylation and transcriptional synergy on complex promoters was first observed for GR [21,24,54,55] and later expanded to other transcription factors including the nuclear receptors AR, MR and PR [6,19,56], and transcription factors like C/EBP, SF1, MITF and ZBP89 [22,57-60]. GRs are modified post-translationally at three consensus SUMO conjugation sites, two in the N-terminus, one in the LBD $[24,54,55]$. Mutation of both N-terminal sites strongly enhances GR-dependent transcription on dual hormone response elements (HREs), but not on the MMTV-LTR [54]. These two N-terminal GR sites, dubbed "synergy control motifs" [21], require an intact receptor LBD and an engaged DBD dimerization interface. Holmstorm et al. [54] propose that stable binding of SUMOylated GR to multiple HREs allows recruitment of inhibitory factors, but that on non-canonical half-site elements such as the MMTV-LTR, SUMOylated GR escape these negative influences [54]. Consistent with these observations, we observe that the single $\mathrm{N}$-terminal PR SUMOylation motif controls transcriptional synergy on multiple PREs but not at a single PRE [6] or the MMTVLTR (Figure 1).

Like GR, AR are SUMOylated at two N-terminal Lys residues and mutation of one (K385) enhances cooperativity on palindromic but not direct-repeat HREs. Callevaert et al. [61] posit that this is a reflection of differing AR dimer conformations on the two types of DNA binding sites. The DBD dimer interface of steroid receptors stabilizes binding to palindromic HREs but this structure forms only after the receptors have bound to DNA [62]. This interface is essential for transcriptional activity on a single HRE, so that mutations in either MR or GR that destabilize it, disrupt receptor/DNA interactions. However, paradoxically these same dimer interface mutations markedly increase synergistic activity of receptors bound to multiple HREs while only modestly increasing DNA binding (62). Mutations in PRs that destabilize the DBD dimer interface also disrupt receptor binding and activity at a single PRE [11], while the same mutations dramatically enhance PR transcriptional activity on promoters containing multiple PREs (PR-B DX; Additional file 1: Figure S2). These mutants are still subject to SUMOylation however (data not shown), suggesting that, as previously reported for GR [54], SUMOylation is upstream of synergy control. Liu et al. (62) postulate that an inhibitory interaction between the N-terminus and the wildtype DBD dimer interface is relieved by DBD mutations, thereby promoting cooperative binding among multimeric receptors and/or coregulatory factors. We speculate that this inhibitory factor is the 97aa SUMO peptide bound at the $\mathrm{N}$-terminus. Its removal, by mutation of the SUMOylation motif or enzymatically with SENP1, relieves the inhibition and permits assembly of higher order PR complexes on DNA.

\section{DeSUMOylation by SENP}

The SENPs deconjugate SUMO-modified proteins and are critical for maintaining physiological ratios of SUMOylated to deSUMOylated substrates. Studies in knockout mice demonstrate that a fine balance of SUMOylation/ deSUMOylation is required for normal embryonic development [29]. This balance may be altered in malignancies. Persistent elevation of SENP1 facilitates the transformation of the normal prostate to a dysplastic state in transgenic mice. Increased SENP expression is observed in malignancies including oncocytic thyroid adenomas, colon and prostate cancers [28,63-66]. Remarkably this control by SUMOylation is maintained despite the fact that usually, $<5 \%$ of target proteins are covalently modified (Figure 2A, for example).

SENP1 stimulates the transcriptional activity of ARs and two different mechanisms have been proposed. Cheng et al. [20] suggest that the transactivating effects of SENP1 do not involve SUMO deconjugation of the receptors, but rather cleavage of SUMO from HDAC1 thereby alleviated its repressive effect on AR activity. In contrast, Kaikkonen et al. [19] demonstrate that effects of SENP1 and SENP2 require intact $S U M O$ acceptor sites in $A R$, indicating that the coactivating effects of the enzymes are directly on the receptors. We show here that both SENP1 and SENP2 stimulate the transcriptional activity of exogenous PR in HeLa cells (Figure 2B), and endogenous PR in T47Dco cells (Additional file 1: Figure S1B). This stimulatory effect is dependent on their enzymatic activity (Figure 2B), requires an intact PR SUMO conjugation site (Figure 3A, B), and functions only at promoters containing multiple PREs 
(Figure 3A, C). To test if SENP1 influences PR activity indirectly, we used the HDAC inhibitor TSA. Inhibition of HDAC activity by TSA did not prevent SENP1 stimulation of wild-type PR (Figure 8B). SUMOylation-deficient PR were similarly affected by TSA, indicating that other mechanisms are responsible for the suppressive effects of SUMOylation on PR activity. This is in agreement with a recent report showing that wild type and SUMOylation deficient AR are similarly influenced by TSA [19]. Taken together we conclude that SENPs target the PR SUMOylation site synergy control function.

\section{PR phosphorylation and SUMOylation}

Both PR SUMOylation and PR phosphorylation are enhanced with similar kinetics by progestin binding to the receptors [18]. However, these two posttranslational protein modification steps appear to be independent of one another. We have shown that K388 SUMOylation of PRs, previously mutated at their MAPK-targeted, progestin-dependent Ser294/344/345 phosphorylation sites, is comparable to SUMOylation of wild-type PRs [6]. On the other hand, activation of MAPK signaling by overexpressing MEKK1 has complex, concentration-dependent effects on PR SUMOylation. At low concentrations, MEKK1 induces ligand-independent PR SUMOylation (Additional file 1: Figure S3B, lanes 3) and increases basal PR-dependent transcription (Figure 6). At high concentrations, MEKK1 suppresses hormone-dependent PR SUMOylation (Additional file 1: Figure S3B, lanes $6 \& 8)$. These contrasting dual activities of MEKK1 suggest that the effects of MAPK on PR SUMOylation are indirect, through alteration of the activity of the general SUMOylation machinery. The molecular mechanisms by which MAPK signaling could indirectly influence PR SUMOylation include changes in the amounts and/or the activities of E3 ligases and cleaving enzymes $[67,68]$. In concert with our conclusions, Kaikkonen et al. [19] recently showed that AR phosphorylation has no effects on AR SUMOylation. Indeed, there are no phosphorylation-dependent SUMOylation motifs in either AR or PR. That PR phosphorylation at S294 does not affect PR SUMOylation is consistent with our data showing that there are no significant differences between the transcriptional activities of wild-type PR and an S294A PR mutant (Figure 6A, B). Qiu et al. [69] have shown similarly robust transcription with a PR S294A mutant. In contrast, Daniel et al. [18] concluded that an association does exist between hormone-dependent PR phosphorylation and PR SUMOylation. The reasons for these differences are unclear but may be related to experimental conditions including use of DNA concentrations for receptor expression at which squelching effects are observed [6].
In contrast to the stimulatory effects of SENP1 on PR activity (Figure 3A), the effect of MAPK signaling on PR transcriptional activity is not related directly to the deSUMOylase effect seen at high concentration (Additional file 1: Figure S3B). First, MEKK1 enhanced hormone independent PR activity (Figure 6A inset and Shen et al. [49]). Second, constitutively active NT-B cannot be SUMOylated, but can still be activated by MEKK1 (Additional file 1: Figure S3A). Third, although SUMOylation has no effect on the MMTV promoter (Figure 1C), MEKK enhances PR dependent activity on this promoter (data not shown). Taken together, our results suggest that the effects of MEKK do not depend on modulation of PR SUMOylation.

\section{Acetylation and SUMOylation}

Acetylation of steroid receptors results in either transcriptional activation or repression, depending on alterations in DNA binding affinities, coregulator recruitment, or hormone responsiveness [16,70-72]. Acetylation and SUMOylation can in theory compete for the same Lys residue of some proteins [73]. In response to hormones, PRs are acetylated at a Lys-rich KxKK motif (aa 638641) conserved in other steroid receptors, and located in the C-terminal hinge region [16]. However, for PR, a Lys to Arg mutation of these residues does not influence N-terminal SUMOylation [16]. We show that SENP1 does not influence the transcriptional activity of DBDLBD (Figure 4B) which contains the acetylation motif (Figure 1A), suggesting dissociation between hinge region acetylation and deSUMOylation.

It has been suggested that SUMOylation represses transcription by recruiting repressors, including HDAC to SUMOylated substrates [46]. However, the transcriptional activities of wild-type and SUMOylation-deficient mutant PRs are both increased by the HDAC inhibitor TSA (Figure 8B), suggesting that other mechanisms are responsible for inhibition of PR activity by SUMOylation. Effects of TSA depend on the concentration used and the cell type analyzed [74,75]. Indeed, low concentrations (50 and $100 \mathrm{nM}$ ) of TSA enhance PR transcriptional activity (Figure 7A) as previously reported [76]. They also promote PR acetylation [16]. However, the effects of TSA on transcription are not related to receptor acetylation since an acetylation-deficient PR-B mutant retains heightened transcriptional activity [16]. On the other hand, at high concentrations (500 and $1000 \mathrm{nM}$ ) TSA markedly inhibits PR transcriptional activity (Figure 7A), and enhances protein stability (Figure 8A, lanes 9, 10). These results are in agreement with studies showing that TSA increases ER acetylation as well as protein stability without affecting ER transcript levels [71]. The inhibitory effect of high TSA levels on PR activity may in part be due to failed ligand 
dependent downregulation (Figure 8A; [49]), and in part to inhibition of coactivator expression and/or assembly. As we show in Figure 7C, overexpression of SRC1 relieves TSA inhibition in a dose dependent manner.

\section{Conclusions}

PRs are major markers in breast cancer. Their presence indicates that a tumor is hormone-dependent and a candidate for endocrine therapies. The role of progesterone in activating these transcription factors is complex, however. After binding PR, progestin agonists and antagonists can have either transcriptional activating or suppressive effects modulated in part by enhancing or suppressing PR SUMOylation $[6,18,31,33,35]$. This study defines the roles of the SUMO-specific SENP proteases and SUMOylation on PR-dependent transcriptional synergy. 1 . We show that deSUMOylation by SENP1 enhances transcriptional synergism in a promoter-specific manner. 2. We also show that SENPs, through their catalytic activity, act at the single K388 PR SUMOylation site, which if mutated eliminates transcriptional synergism by SENPs. 3. The enzymes can act only on hormone-bound full-length PRs and increase the ligand sensitivity of the receptors. 4. SUMOylation effects on PR transcriptional synergism are dissociable from receptor phosphorylation, SRC-1 coactivation or recruitment of HDACs to the promoter. We conclude that reversible SUMOylation/deSUMOylation of a minor PR protein subpopulation tightly controls the overall transcriptional activity of the receptors at complex synthetic promoters. Of note we previously showed a requirement for PR SUMOylation to transrepress ER thereby altering tumor responses to estrogens [33]. Taken together, our data suggest that the PR SUMO modification pathway critically modifies the response of a tumor to estrogens, progestins and antiprogestins-hormones that are major therapeutics for breast cancers.

\section{Methods \\ Plasmids}

The expression plasmids pSG5 hPR, encoding human PR-B and HEGO, encoding human ER, cloned into pSG5 were a gift of P. Chambon (Strasbourg, France). Cloning of pSG5 hPR1 K388R, pSG5 hPR1 S294/344/ 345A, pSG5 NT-B, pSG5 hPR1 R606A (PR-B DNA dimerization mutant was a gift of B. Jacobsen), pCMV5MEKK1 and pSG5 DBD-LBD were described previously $[4,11,17,33]$. Wild type pEGFP-SUMO-1 was a gift of J. Palvimo and O. Janne (University of Helsinki, Helsinki, Finland). pCR3.1-SRC-1e was a gift of B. O'Malley (Baylor College of Medicine, Houston, TX). ERE ${ }_{2}-\mathrm{Luc}_{\text {, }} \mathrm{PRE}_{2}-$ Luc and MMTV-Luc reporter plasmids were described previously [4]. Flag-SENP1, Flag-SENP1 mutant (R630L,
K631M) and Flag-SENP2 were gifts of E. Yeh (M. D. Anderson, Houston, TX).

\section{Transcription assays}

HeLa cells were plated in minimum Eagle's medium containing 5\% FBS (twice charcoal-stripped for experiments with full-length PR or DBD-LBD) at a density of $1.2 \times$ $10^{5}$ cells per $60 \mathrm{~mm}$ dish, 1 day prior to transfection. Cells were transfected by calcium phosphate co-precipitation [42] with concentrations of expression vectors indicated in the figures. Reporter plasmids were added at $2 \mu \mathrm{g} /$ dish. SV40-Renilla luciferase was added as an internal control at $20 \mathrm{ng} /$ dish. Twenty four hours later, cells expressing LBD-containing constructs were washed and incubated 24 hrs with the synthetic progestin R5020 (Sigma Chemical Co., St. Louis, MO) at final concentrations indicated in the figures. Control cells received ethanol only. Cells were collected in $150 \mu$ l lysis buffer (Promega), and $50 \mu \mathrm{l}$ were analyzed on a dual luminometer [42]. Results were normalized to Renilla luciferase activity and expressed as indicated in the figures. Replicate experiments were done in duplicate.

\section{Immunoblotting}

Whole cell extracts were prepared from HeLa cells transiently transfected with PR expression vectors as described [33]. Cells were treated with 10 nM R5020 and/or Trichostatin A (TSA). Lysates containing equal protein concentrations were resolved by SDS-PAGE, transferred to nitrocellulose, and probed with anti-PR PgR1294 (DakoCytomation) or anti- $\beta$-actin AC-74 (Sigma) monoclonal antibodies. Bands were detected by enhanced chemiluminescence (PerkinElmer Life Sciences). For PR SUMOylation, HeLa cells cotransfected with PR and GFP-tagged SUMO-1 were collected in PBS containing $20 \mathrm{mM} \mathrm{N}$-ethylmaleimide, and cell extracts were prepared in $50 \mathrm{mM}$ Tris- $\mathrm{HCl}(\mathrm{pH} 7.8)$, $150 \mathrm{mM} \mathrm{NaCl}, 5 \mathrm{mM}$ EDTA, $15 \mathrm{mM}$ dithiothreitol, a protease inhibitor mixture (Roche Molecular Biochemicals), and $20 \mathrm{mM} \mathrm{N}$-ethylmaleimide. The expressed proteins were resolved on SDS-PAGE, and conjugated protein was detected by immunoblotting with PgR1294.

\section{Statistical analysis}

Prism GraphPad software version 4 (GraphPad Software Inc. La Jolla, CA). was used to determine least-squares best fit of the experimental data to the theoretical doseresponse curve. All values represent at least three independent experiments and are expressed as the means \pm SD. Data sets were analyzed with GraphPad Prism 4 Statistical significance was determined by two-tailed unpaired student's $t$ test, and the differences were considered statistically significant at a $\mathrm{P}$ value of 0.05 . 


\section{Additional material}

\section{Additional file 1: Figure S1. A) DeSUMOylation of PR by SENP1}

depends on its catalytic activity. HeLa cells were transiently transfected with expression vectors encoding wild type PR-B together with a GFPSUMO-1 expression vector (+), and wild type or mutant (m) SENP1. Cells were treated 24 hrs without (-) or with (+) 10 nM R5020. Western blot analysis was performed on cell extracts probed with the anti-PR1294 monoclonal antibody or anti -actin control. B) SENP1 enhances PR-B activity in T47D breast cancer cells. PR-negative T47D-Y breast cancer cells stably expressing PR-B were transfected with the PRE2-Luc reporter plasmid in the presence of pSV40-Renilla as internal control along with increasing amount (20-1000 ng) of SENP1 expression vector, or an empty vector control (-). Cells were treated without (-) or with (+) 10 nM R5020 for 24 hrs before being assayed for luciferase activity. C) SENP1 enhances transcription by the partial agonist RU486. HeLa cells were transfected with $2 \mathrm{~g}$ of PRE2-luciferase reporters together with $50 \mathrm{ng}$ of a PR-B expression vector and Renilla-Luc as an internal control in the presence or absence of $100 \mathrm{ng}$ SENP1 or SENP1m expression vectors. The cells were treated for $24 \mathrm{hrs}$ with the agonist R5020 (10 nM), partial agonist RU486 (100 nM), or the pure antagonist ZK98299 (100 nM) then harvested and lysed. The extracts were assayed for luciferase activities as in Figure 1. Figure S2. The PR DBD dimerization interface is necessary for effective synergy control. HeLa cells were transfected with $2 \mathrm{~g}$ of PRE2-luciferase reporters together with $50 \mathrm{ng}$ of a wild type PR - $B$, the PR-B K388R SUMOylation deficient, or a PR-B DBD dimerization mutant (PR-B DX) expression vector and Renilla-Luc as an internal control in the presence or absence of $100 \mathrm{ng}$ SENP1 expression vectors. The cells were treated for 24 hrs with the agonist R5020 (10 nM), then harvested and lysed. The extracts were assayed for luciferase activities as in Figure 1. Figure S3. A) The stimulatory effect of MEKK1 on PR-B transcriptional activity is LBD and hormone independent. HeLa cells were transfected with $2 \mathrm{~g}$ of PRE2-luciferase reporters together with 500 ng of NTB-DBD, a constitutively active PR N-terminal expression vector in the presence of pSV40-Renilla as internal control along with increasing amount (5-200 ng) of constitutively active MEKK1 expression vector, or an empty vector control (-). The extracts were assayed for luciferase activities as in Figure 1. B) Concentration dependent effect of MEKK1 on PR SUMOylation. HeLa cells were transiently transfected with expression vectors encoding wild type PR-B together with a GFP-SUMO-1 expression vector $(+)$ in the absence $(-)$ or presence of increasing amount of MEKK1 expression vector. Cells were treated 24 hrs without (-) or with (+) $10 \mathrm{nM}$ R5020. Western blot analysis was performed on cell extracts probed with the anti-PR1294 monoclonal antibody or anti -actin control.

\section{Abbreviations}

AF: Activation function; AR: Androgen receptors; BUS: PR-B-upstream segment; DBD: DNA binding domain; ER: Estrogen receptors; GFP: Green fluorescent protein; GR: Glucocorticoid receptors; HDAC: Histone deacetylase; LBD: Ligand binding domain; MMTV: Mouse mammary tumor virus; MR: Mineralocorticoid receptors; NEM: N-ethylmaleimide; NR: Nuclear receptors; NT-B: N-terminal region of PR-B; PIAS: Protein inhibitor of activated transducer and activator of transcription; PR: Progesterone receptors; PRE: Progesterone response element; SENP: SUMO specific protease; SRC: Steroid receptor coactivator; SUMO: Small ubiquitin like modifier; TSA: Trichostatin A

\section{Acknowledgements}

We greatly appreciate the gifts of reagents from several colleagues described in Experimental Procedures. We thank Michelle Dudevoir for technical assistance. These studies were funded by the NIH, NCI CA02686933; the National Foundation for Cancer Research; the Breast Cancer Research Foundation; and the Avon Foundation for Women.

\section{Author details}

'Division of Endocrinology, Department of Medicine, Anschutz Medical Campus, University of Colorado, Aurora, CO 80045, USA. ${ }^{2}$ Department of Pathology, Anschutz Medical Campus, University of Colorado, Aurora, CO
80045, USA. ${ }^{3}$ University of Colorado AMC, Mail Stop 8106, 12801 East 17th Avenue, Aurora, CO 80045, USA.

\section{Authors' contributions}

$\mathrm{HA}-\mathrm{H}$ was involved in all of the experimental and theoretical work. $\mathrm{HA}-\mathrm{H}$ and $\mathrm{KBH}$ participated in the design of the experiments. $\mathrm{HA}-\mathrm{H}$ and $\mathrm{KBH}$ wrote the manuscript. All authors have read and approved the final manuscript.

\section{Competing interests}

The authors declare that they have no competing interests.

Received: 22 November 2011 Accepted: 22 March 2012

Published: 22 March 2012

\section{References}

1. Richer JK, Jacobsen BM, Manning NG, Abel MG, Wolf DM, Horwitz KB: Differential gene regulation by the two progesterone receptor isoforms in human breast cancer cells. J Biol Chem 2002, 277(7):5209-5218.

2. Wei LL, Gonzalez-Aller C, Wood WM, Miller LA, Horwitz KB: $5^{\prime}$ Heterogeneity in human progesterone receptor transcripts predicts a new amino-terminal truncated " $\mathrm{C}$ "-receptor and unique A-receptor messages. Mol Endocrinol 1990, 4(12):1833-1840.

3. Sartorius CA, Groshong SD, Miller LA, Powell RL, Tung L, Takimoto GS, Horwitz KB: New T47D breast cancer cell lines for the independent study of progesterone B- and A-receptors: only antiprogestin-occupied Breceptors are switched to transcriptional agonists by CAMP. Cancer Res 1994, 54(14):3868-3877.

4. Tung L, Abdel-Hafiz H, Shen T, Harvell DM, Nitao LK, Richer JK, Sartorius CA, Takimoto GS, Horwitz KB: Progesterone receptors (PR)-B and -A regulate transcription by different mechanisms: AF-3 exerts regulatory control over coactivator binding to PR-B. Mol Endocrinol 2006, 20(11):2656-2670.

5. Sheridan PL, Francis MD, Horwitz KB: Synthesis of human progesterone receptors in T47D cells. Nascent A- and B-receptors are active without a phosphorylation-dependent post-translational maturation step. J Biol Chem 1989, 264(12):7054-7058.

6. Abdel-Hafiz H, Dudevoir ML, Horwitz KB: Mechanisms underlying the control of progesterone receptor transcriptional activity by SUMOylation J Biol Chem 2009, 284(14):9099-9108.

7. Owen Gl, Richer JK, Tung L, Takimoto G, Horwitz KB: Progesterone regulates transcription of the p21(WAF1) cyclin-dependent kinase inhibitor gene through Sp1 and CBP/p300. J Biol Chem 1998, 273(17):10696-10701.

8. Kalkhoven E, Beraldi E, Panno ML, De Winter JP, Thijssen JH, Van Der Burg B: Growth inhibition by anti-estrogens and progestins in TGF-betaresistant and -sensitive breast-tumor cells. Int J Cancer 1996, 65(5):682-687.

9. Madauss KP, Grygielko ET, Deng SJ, Sulpizio AC, Stanley TB, Wu C, Short SA, Thompson SK, Stewart EL, Laping NJ, et al: A structural and in vitro characterization of asoprisnil: a selective progesterone receptor modulator. Mol Endocrinol 2007, 21(5):1066-1081.

10. Subtil-Rodriguez A, Millan-Arino L, Quiles I, Ballare C, Beato M, Jordan A: Progesterone induction of the 11 beta-hydroxysteroid dehydrogenase type 2 promoter in breast cancer cells involves coordinated recruitment of STAT5A and progesterone receptor to a distal enhancer and polymerase tracking. Mol Cell Biol 2008, 28(11):3830-3849.

11. Jacobsen BM, Jambal P, Schittone SA, Horwitz KB: ALU repeats in promoters are position-dependent co-response elements (coRE) that enhance or repress transcription by dimeric and monomeric progesterone receptors. Mol Endocrinol 2009, 23(7):989-1000.

12. Onate $S A$, Prendergast $P$, Wagner JP, Nissen $M$, Reeves $R$, Pettijohn DE, Edwards DP: The DNA-bending protein HMG-1 enhances progesterone receptor binding to its target DNA sequences. Mol Cell Biol 1994, 14(5):3376-3391.

13. Connaghan-Jones KD, Heneghan AF, Miura MT, Bain DL: Thermodynamic analysis of progesterone receptor-promoter interactions reveals a molecular model for isoform-specific function. Proc Natl Acad Sci USA 2007, 104(7):2187-2192.

14. Heneghan AF, Connaghan-Jones KD, Miura MT, Bain DL: Coactivator assembly at the promoter: efficient recruitment of SRC2 is coupled to cooperative DNA binding by the progesterone receptor. Biochemistry 2007, 46(39):11023-11032. 
15. Faus $\mathrm{H}$, Haendler B: Post-translational modifications of steroid receptors. Biomed Pharmacother 2006, 60(9):520-528.

16. Daniel AR, Gaviglio AL, Czaplicki LM, Hillard CJ, Housa D, Lange CA: The progesterone receptor hinge region regulates the kinetics of transcriptional responses through acetylation, phosphorylation, and nuclear retention. Mol Endocrinol 2010, 24(11):2126-2138.

17. Lange CA, Shen T, Horwitz KB: Phosphorylation of human progesterone receptors at serine-294 by mitogen-activated protein kinase signals their degradation by the 265 proteasome. Proc Natl Acad Sci USA 2000, 97(3):1032-1037.

18. Daniel AR, Faivre EJ, Lange CA: Phosphorylation-dependent antagonism of sumoylation derepresses progesterone receptor action in breast cancer cells. Mol Endocrinol 2007, 21(12):2890-2906.

19. Kaikkonen S, Jaaskelainen T, Karvonen U, Rytinki MM, Makkonen H, Gioeli D, Paschal BM, Palvimo JJ: SUMO-specific protease 1 (SENP1) reverses the hormone-augmented SUMOylation of androgen receptor and modulates gene responses in prostate cancer cells. Mol Endocrinol 2009, 23(3):292-307.

20. Cheng J, Wang D, Wang Z, Yeh ET: SENP1 enhances androgen receptordependent transcription through desumoylation of histone deacetylase 1. Mol Cell Biol 2004, 24(13):6021-6028.

21. Iniguez-Lluhi JA, Pearce D: A common motif within the negative regulatory regions of multiple factors inhibits their transcriptional synergy. Mol Cell Biol 2000, 20(16):6040-6050.

22. Molvaersmyr AK, Saether T, Gilfillan S, Lorenzo PI, Kvaloy H, Matre V, Gabrielsen OS: A SUMO-regulated activation function controls synergy of c-Myb through a repressor-activator switch leading to differential p300 recruitment. Nucleic Acids Res 2010, 38(15):4970-4984.

23. Holmstrom S, Van Antwerp ME, Iniguez-Lluhi JA: Direct and distinguishable inhibitory roles for SUMO isoforms in the control of transcriptional synergy. Proc Natl Acad Sci USA 2003, 100(26):15758-15763.

24. Tian S, Poukka H, Palvimo JJ, Janne OA: Small ubiquitin-related modifier-1 (SUMO-1) modification of the glucocorticoid receptor. Biochem J 2002, 367(Pt 3):907-911.

25. Palvimo JJ: PIAS proteins as regulators of small ubiquitin-related modifier (SUMO) modifications and transcription. Biochem Soc Trans 2007, 35(Pt 6):1405-1408.

26. Poukka H, Aarnisalo P, Karvonen U, Palvimo JJ, Janne OA: Ubc9 interacts with the androgen receptor and activates receptor-dependent transcription. J Biol Chem 1999, 274(27):19441-19446.

27. Kotaja N, Aittomaki S, Silvennoinen O, Palvimo JJ, Janne OA: ARIP3 (androgen receptor-interacting protein 3 ) and other PIAS (protein inhibitor of activated STAT) proteins differ in their ability to modulate steroid receptor-dependent transcriptional activation. Mol Endocrinol 2000, 14(12):1986-2000.

28. Bawa-Khalfe T, Yeh ET: SUMO Losing Balance: SUMO Proteases Disrupt SUMO Homeostasis to Facilitate Cancer Development and Progression. Genes Cancer 2010, 1(7):748-752.

29. Bawa-Khalfe T, Yeh ET: The in vivo functions of desumoylating enzymes. Subcell Biochem 2011, 54:170-183.

30. Poukka H, Karvonen U, Janne OA, Palvimo JJ: Covalent modification of the androgen receptor by small ubiquitin-like modifier 1 (SUMO-1). Proc Natl Acad Sci USA 2000, 97(26):14145-14150.

31. Jones MC, Fusi L, Higham JH, Abdel-Hafiz H, Horwitz KB, Lam EW, Brosens JJ: Regulation of the SUMO pathway sensitizes differentiating human endometrial stromal cells to progesterone. Proc Natl Acad Sci USA 2006, 103(44):16272-16277

32. Man JH, Li HY, Zhang PJ, Zhou T, He K, Pan X, Liang B, Li AL, Zhao J, Gong WL, et al: PIAS3 induction of PRB sumoylation represses PRB transactivation by destabilizing its retention in the nucleus. Nucleic Acids Res 2006, 34(19):5552-5566.

33. Abdel-Hafiz H, Takimoto GS, Tung L, Horwitz KB: The inhibitory function in human progesterone receptor $\mathrm{N}$ termini binds SUMO-1 protein to regulate autoinhibition and transrepression. J Biol Chem 2002, 277(37):33950-33956

34. Takimoto GS, Tung L, Abdel-Hafiz H, Abel MG, Sartorius CA, Richer JK, Jacobsen BM, Bain DL, Horwitz KB: Functional properties of the N-terminal region of progesterone receptors and their mechanistic relationship to structure. J Steroid Biochem Mol Biol 2003, 85(2-5):209-219.

35. Chauchereau A, Amazit L, Quesne M, Guiochon-Mantel A, Milgrom E: Sumoylation of the progesterone receptor and of the steroid receptor coactivator SRC-1. J Biol Chem 2003, 278(14):12335-12343.
36. Zhang PJ, Zhao J, Li HY, Man JH, He K, Zhou T, Pan X, Li AL, Gong WL, Jin $B F$, et al: CUE domain containing 2 regulates degradation of progesterone receptor by ubiquitin-proteasome. EMBO J 2007, 26(7):1831-1842.

37. Wilson MA, Ricci AR, Deroo BJ, Archer TK: The histone deacetylase inhibitor trichostatin $A$ blocks progesterone receptor-mediated transactivation of the mouse mammary tumor virus promoter in vivo. J Biol Chem 2002, 277(17):15171-15181.

38. Gowland PL, Buetti E: Mutations in the hormone regulatory element of mouse mammary tumor virus differentially affect the response to progestins, androgens, and glucocorticoids. Mol Cell Biol 1989, 9(9):3999-4008.

39. Connaghan-Jones KD, Heneghan AF, Miura MT, Bain DL: Thermodynamic dissection of progesterone receptor interactions at the mouse mammary tumor virus promoter: monomer binding and strong cooperativity dominate the assembly reaction. J Mol Biol 2008, 377(4):1144-1160.

40. Lim CS, Baumann CT, Htun H, Xian W, Irie M, Smith CL, Hager GL: Differential localization and activity of the A- and B-forms of the human progesterone receptor using green fluorescent protein chimeras. Mol Endocrinol 1999, 13(3):366-375.

41. Sartorius CA, Tung L, Takimoto GS, Horwitz KB: Antagonist-occupied human progesterone receptors bound to DNA are functionally switched to transcriptional agonists by CAMP. J Biol Chem 1993, 268(13):9262-9266.

42. Tung L, Shen T, Abel MG, Powell RL, Takimoto GS, Sartorius CA, Horwitz KB: Mapping the unique activation function 3 in the progesterone Breceptor upstream segment. Two LXXLL motifs and a tryptophan residue are required for activity. J Biol Chem 2001, 276(43):39843-39851.

43. Takimoto GS, Hovland AR, Tasset DM, Melville MY, Tung L, Horwitz KB: Role of phosphorylation on DNA binding and transcriptional functions of human progesterone receptors. J Biol Chem 1996, 271(23):13308-13316.

44. Weigel NL, Bai W, Zhang Y, Beck CA, Edwards DP, Poletti A: Phosphorylation and progesterone receptor function. I Steroid Biochem Mol Biol 1995, 53(1-6):509-514.

45. Knotts TA, Orkiszewski RS, Cook RG, Edwards DP, Weigel NL: Identification of a phosphorylation site in the hinge region of the human progesterone receptor and additional amino-terminal phosphorylation sites. J Biol Chem 2001, 276(11):8475-8483.

46. Yang SH, Sharrocks AD: SUMO promotes HDAC-mediated transcriptional repression. Mol Cell 2004, 13(4):611-617.

47. Toth KF, Knoch TA, Wachsmuth M, Frank-Stohr M, Stohr M, Bacher CP, Muller G, Rippe K: Trichostatin A-induced histone acetylation causes decondensation of interphase chromatin. J Cell Sci 2004, 117(Pt 18):4277-4287.

48. Welsbie DS, Xu J, Chen Y, Borsu L, Scher HI, Rosen N, Sawyers CL: Histone deacetylases are required for androgen receptor function in hormonesensitive and castrate-resistant prostate cancer. Cancer Res 2009, 69(3):958-966.

49. Shen T, Horwitz KB, Lange CA: Transcriptional hyperactivity of human progesterone receptors is coupled to their ligand-dependent downregulation by mitogen-activated protein kinase-dependent phosphorylation of serine 294. Mol Cell Biol 2001, 21(18):6122-6131.

50. Stielow B, Sapetschnig A, Kruger I, Kunert N, Brehm A, Boutros M, Suske G: Identification of SUMO-dependent chromatin-associated transcriptional repression components by a genome-wide RNAi screen. Mol Cell 2008, 29(6):742-754.

51. Lin DY, Huang YS, Jeng JC, Kuo HY, Chang CC, Chao TT, Ho CC, Chen YC, Lin TP, Fang H, et al: Role of SUMO-interacting motif in Daxx SUMO modification, subnuclear localization, and repression of sumoylated transcription factors. Mol Cell 2006, 24(3):341-354.

52. Lee MB, Lebedeva LA, Suzawa M, Wadekar SA, Desclozeaux M, Ingraham HA: The DEAD-box protein DP103 (Ddx20 or Gemin-3) represses orphan nuclear receptor activity via SUMO modification. Mol Cell Biol 2005, 25(5):1879-1890.

53. Rosendorff A, Sakakibara S, Lu S, Kieff E, Xuan Y, DiBacco A, Shi Y, Gill G: NXP-2 association with SUMO-2 depends on lysines required for transcriptional repression. Proc Natl Acad Sci USA 2006, 103(14):5308-5313.

54. Holmstrom SR, Chupreta S, So AY, Iniguez-Lluhi JA: SUMO-mediated inhibition of glucocorticoid receptor synergistic activity depends on stable assembly at the promoter but not on DAXX. Mol Endocrinol 2008, 22(9):2061-2075. 
55. Le Drean Y, Mincheneau N, Le Goff P, Michel D: Potentiation of glucocorticoid receptor transcriptional activity by sumoylation. Endocrinology 2002, 143(9):3482-3489.

56. Tallec LP, Kirsh O, Lecomte MC, Viengchareun S, Zennaro MC, Dejean A, Lombes M: Protein inhibitor of activated signal transducer and activator of transcription 1 interacts with the N-terminal domain of mineralocorticoid receptor and represses its transcriptional activity: implication of small ubiquitin-related modifier 1 modification. Mol Endocrinol 2003, 17(12):2529-2542.

57. Khanna-Gupta A: Sumoylation and the function of CCAAT enhancer binding protein alpha (C/EBP alpha). Blood Cells Mol Dis 2008, 41(1):77-81.

58. Miller AJ, Levy C, Davis IJ, Razin E, Fisher DE: Sumoylation of MITF and its related family members TFE3 and TFEB. J Biol Chem 2005, 280(1):146-155.

59. Chupreta S, Brevig H, Bai L, Merchant JL, Iniguez-Lluhi JA: Sumoylationdependent control of homotypic and heterotypic synergy by the Kruppel-type zinc finger protein ZBP-89. J Biol Chem 2007, 282(50):36155-36166.

60. Komatsu T, Mizusaki H, Mukai T, Ogawa H, Baba D, Shirakawa M, Hatakeyama S, Nakayama Kl, Yamamoto H, Kikuchi A, et al: Small ubiquitinlike modifier 1 (SUMO-1) modification of the synergy control motif of Ad4 binding protein/steroidogenic factor 1 (Ad4BP/SF-1) regulates synergistic transcription between Ad4BP/SF-1 and Sox9. Mol Endocrinol 2004, 18(10):2451-2462.

61. Callewaert L, Verrijdt G, Haelens A, Claessens F: Differential effect of small ubiquitin-like modifier (SUMO)-ylation of the androgen receptor in the control of cooperativity on selective versus canonical response elements. Mol Endocrinol 2004, 18(6):1438-1449.

62. Liu W, Wang J, Yu G, Pearce D: Steroid receptor transcriptional synergy is potentiated by disruption of the DNA-binding domain dimer interface. Mol Endocrinol 1996, 10(11):1399-1406.

63. Yeh ET: SUMOylation and De-SUMOylation: wrestling with life's processes. J Biol Chem 2009, 284(13):8223-8227.

64. Cheng J, Bawa T, Lee P, Gong L, Yeh ET: Role of desumoylation in the development of prostate cancer. Neoplasia 2006, 8(8):667-676.

65. Jacques C, Baris O, Prunier-Mirebeau D, Savagner F, Rodien P, Rohmer V, Franc B, Guyetant S, Malthiery Y, Reynier P: Two-step differential expression analysis reveals a new set of genes involved in thyroid oncocytic tumors. J Clin Endocrinol Metab 2005, 90(4):2314-2320.

66. Xu Y, Li J, Zuo Y, Deng J, Wang LS, Chen GQ: SUMO-specific protease 1 regulates the in vitro and in vivo growth of colon cancer cells with the upregulated expression of CDK inhibitors. Cancer Lett 2011, 309(1):78-84.

67. Yang SH, Sharrocks AD: PIASxalpha differentially regulates the amplitudes of transcriptional responses following activation of the ERK and p38 MAPK pathways. Mol Cell 2006, 22(4):477-487.

68. Liu B, Yang Y, Chernishof V, Loo RR, Jang H, Tahk S, Yang R, Mink S, Shultz D, Bellone CJ, et al: Proinflammatory stimuli induce IKKalphamediated phosphorylation of PIAS1 to restrict inflammation and immunity. Cell 2007, 129(5):903-914.

69. Qiu M, Lange CA: MAP kinases couple multiple functions of human progesterone receptors: degradation, transcriptional synergy, and nuclear association. J Steroid Biochem Mol Biol 2003, 85(2-5):147-157.

70. Fu M, Rao M, Wang C, Sakamaki T, Wang J, Di Vizio D, Zhang X, Albanese C, Balk S, Chang C, et al: Acetylation of androgen receptor enhances coactivator binding and promotes prostate cancer cell growth. Mol Cell Biol 2003, 23(23):8563-8575.

71. Kim MY, Woo EM, Chong YT, Homenko DR, Kraus WL: Acetylation of estrogen receptor alpha by p300 at lysines 266 and 268 enhances the deoxyribonucleic acid binding and transactivation activities of the receptor. Mol Endocrinol 2006, 20(7):1479-1493.

72. Kim Y, Sun Y, Chow C, Pommier YG, Simons SS Jr: Effects of acetylation, polymerase phosphorylation, and DNA unwinding in glucocorticoid receptor transactivation. J Steroid Biochem Mol Biol 2006, 100(1-3):3-17.

73. Bouras T, Fu M, Sauve AA, Wang F, Quong AA, Perkins ND, Hay RT, Gu W, Pestell RG: SIRT1 deacetylation and repression of p300 involves lysine residues 1020/1024 within the cell cycle regulatory domain 1. J Biol Chem 2005, 280(11):10264-10276.

74. Wood JP, Chidlow G, Tran T, Crowston JG, Casson RJ: A comparison of differentiation protocols for RGC-5 cells. Invest Ophthalmol Vis Sci 2010 51(7):3774-3783

75. Alao JP, Lam EW, Ali S, Buluwela L, Bordogna W, Lockey P, Varshochi R, Stavropoulou AV, Coombes RC, Vigushin DM: Histone deacetylase inhibitor trichostatin A represses estrogen receptor alpha-dependent transcription and promotes proteasomal degradation of cyclin D1 in human breast carcinoma cell lines. Clin Cancer Res 2004, 10(23):8094-8104.

76. Jenster G, Spencer TE, Burcin MM, Tsai SY, Tsai MJ, O'Malley BW: Steroid receptor induction of gene transcription: a two-step model. Proc Nat/ Acad Sci USA 1997, 94(15):7879-7884.

doi:10.1186/1471-2199-13-10

Cite this article as: Abdel-Hafiz and Horwitz: Control of progesterone receptor transcriptional synergy by SUMOylation and deSUMOylation. BMC Molecular Biology 2012 13:10.

\section{Submit your next manuscript to BioMed Central and take full advantage of:}

- Convenient online submission

- Thorough peer review

- No space constraints or color figure charges

- Immediate publication on acceptance

- Inclusion in PubMed, CAS, Scopus and Google Scholar

- Research which is freely available for redistribution 